\title{
REPRODUCCIÓN DE IDENTIDAD VIRTUAL DE GÉNERO EN LA INTERACCIÓN DOCENTES-ALUMNADO. UN ESTUDIO DE CASO
}

REPRODUCTION OF VIRTUAL GENDER IDENTITY IN THE TEACHER-STUDENT INTERACTION. A CASE STUDY

\section{Volumen 15, Número 2}

Mayo - Agosto

pp. 1-27

Angélica Riquelme Arredondo Josefina Quintero Corzo

Revista indizada en REDALYC, $\underline{\text { SCIELO }}$

Revista distribuida en las bases de datos:

CATÁLOGO DE LATINDEX, IRESIE, CLASE, DIALNET, DOAJ, E-REVIST@S, SHERPA/ROMEO, QUALIS, MIAR

Revista registrada en los directorios:

ULRICH'S, REDIE, RINACE, OEI, MAESTROTECA, PREAL, CLACSO 


\title{
REPRODUCCIÓN DE IDENTIDAD VIRTUAL DE GÉNERO EN LA INTERACCIÓN DOCENTES-ALUMNADO. UN ESTUDIO DE CASO REPRODUCTION OF VIRTUAL GENDER IDENTITY IN THE TEACHER-STUDENT INTERACTION. A CASE STUDY
}

\author{
Angélica Riquelme Arredondo ${ }^{1}$ \\ Josefina Quintero Corzo ${ }^{2}$
}

\begin{abstract}
Resumen: Este artículo es producto de una investigación bajo enfoque de estudio de caso, que pretendió un análisis sobre educación y género, develando la existencia de discriminación por parte de docentes hacia el alumnado mediante la reproducción de la identidad virtual en la interacción observada en aulas de enseñanza secundaria. Se realizó una profundización de la dimensión teórica, en el contexto del grupo de investigación Educación y Pedagogía: saberes, imaginarios e intersubjetividades del Doctorado en Niñez y Juventud CINDE U. de Manizales, estudiándose la dimensión de género, entendida como la normatividad que impone dos sexos dicotómicos que, construyen a los hombres y a las mujeres desde una matriz heterosexual. Metodológicamente se procuró delinear un camino interpretativo que no solamente arrojara cifras sino que nos permitiera comprender los significados. Se entrevistó a estudiantes y sus docentes en distintas asignaturas de dos colegios particulares de la comuna de Ñuñoa en Santiago de Chile. Dentro de los resultados emerge claramente el trato desigual, que reciben estudiantes por parte de docentes, quienes aceptan las diferencias que trazan en su trato y las justifican caracterizándolas de naturales, por su parte el estudiantado, nota el trato diferencial por parte de éstos, sin embargo, no con la naturalización que se observa en la situación de aula. Se concluye con estos casos, que existe desigualdad en el trato hacia los géneros y tanto estudiantes como docentes contribuyen conscientemente a reproducirla socioculturalmente.
\end{abstract}

Palabras clave: DISCRIMINACIÓN DE GÉNERO, ESTIGMATIZACIÓN, NATURALIZACIÓN, VIOLENCIA SIMBÓLICA, IDENTIDAD SOCIAL VIRTUAL DE GÉNERO, CHILE

\begin{abstract}
This paper results from a case study of gender and education in high school classrooms, which uncovered episodes of gender discrimination from the teachers to the students by their reproduction of virtual gender identity. The theoretical aspects of the paper were developed within the research group Educación y Pedagogía: saberes, imaginarios e intersubjetividades del Doctorado en Niñez y Juventud CINDE U. de Manizales, and the gender dimension was understood as the set of norms that impose two dichotomist sexes, building women and men from a heterosexual matrix. We followed an interpretative path so as to obtain, in addition to figures, an understanding of meanings. We interviewed students and teachers of different courses in two private schools of Nuñoa County in Santiago de Chile. The unequal treatment of the teachers towards the students was apparent. The former recognize the differences and justify them as "natural," while the latter notice the biases but tend to adopt a superficial view of the issues. We conclude that inequality exists and that the actors consciously contribute to reproduce it, revealing the prevalence of a male-chauvinistic social view.
\end{abstract}

Key words: GENDER DISCRIMINATION, STIGMATIZATION, NATURALIZATION, SYMBOLIC VIOLENCE, VIRTUAL SOCIAL GENDER IDENTITY, CHILE

\footnotetext{
Académica Departamento de Educación Universidad de Chile. Alumna del Doctorado Niñez y Juventud, Universidad de ManizalesCINDE Colombia. Dirección electrónica: ariquelme@uchile.cl

2 Profesora Titular Universidad de Caldas, Colombia. Ex-Decana Facultad de Artes y Humanidades. Doctora en Educación de la Universidad de Antioquia, Colombia. Dirección electrónica: josefina.quinterocj@ucladas.edu.co
}

Artículo recibido: 5 de agosto, 2014

Enviado a corrección: 16 de febrero, 2015

Aprobado: 20 de abril, 2015 
"Todo indica que la segmentación educacional con perspectivas profesionales comienzan claramente desde la secundaria". (Rossetti).

\section{Introducción}

Dentro de nuestra sociedad hay ciertas características, funciones e incluso gustos que le son propias a tal o cual género, estas identidades sociales virtuales de género, que en su mayoría responden al constructo social imperante, pasan a entenderse como características naturales de las mujeres $u$ hombres.

La situación de poder y traspaso de conocimiento que representa la interacción entre los docentes y estudiantes, simboliza uno de los momentos más interesantes en el sistema escolar, donde por ejemplo, se genera y prolonga la situación de adopción de estereotipos que más a delante se describen. En la sociedad actual, la institución educativa representa una verdad o más bien, tiene el poder para traspasar "verdades" de generación en generación, estas transferencias, se realizan en base a la autoridad que sostienen los pedagogos y que deriva de lo que se denomina, institución legítima, es decir, la escuela. De éste modo, la autoridad y legitimidad de la escuela se desprende del medio social mismo, el punto social de partida del clásico análisis de la educación como reproducción, y una de las más ricas colaboraciones en el área (Bourdieu y Passeron, 1996).

Este estudio de caso pretende conocer cómo se reproduce la identidad virtual de género en las interacciones de docentes y estudiantes en las aulas y cuál es el significado que le atribuyen los actores a este fenómeno. El problema de investigación, se abordó desde el intento de comprender el significado que éstos otorgaron a dos procesos: (a) la reproducción de la violencia simbólica, en una sociedad donde impera la dominación masculina y que hace uso de las instituciones de la educación para conservar el orden social; (b) la estigmatización, es decir, la sanción generada por el alejamiento de lo que se concibe como identidad femenina o masculina

En virtud de lo anterior, se indagó en los significados otorgados por el cuerpo docente y el alumnado en la interacción de clase en los cursos (del plan común de formación de enseñanza media y electiva) físico-química, químico-biológico y matemático-físico en los segundos medios en dos comunidades educativas.

Cabe destacar que se está consciente que la presente investigación es sólo una pequeña ventana a la arquitectura compleja que construyen las intersecciones de género y educación en Chile, pero que sin duda aporta a la discusión de la presente temática. 


\section{Antecedentes}

De acuerdo con la última Encuesta Nacional de Juventud de Chile, un 53,6\% de los y las jóvenes se encuentran cursando la enseñanza media. Al desagregar dicha información de acuerdo a la variable "sexo", podemos darnos cuenta que no existen grandes diferencias de acceso entre hombres y mujeres: de la población que asiste a educación secundaria, un $51,7 \%$ está conformada por hombres y un $55,5 \%$ por mujeres. (Instituto Nacional de Juventud, 2012). A pesar de la mínima diferencia, se observa una mayor participación femenina.

Para CEPAL (Comisión Económica para América Latina y el Caribe), las expectativas educativas y laborales se desarrollan en la adolescencia (CEPAL, 2014). Es por esta razón, que la educación secundaria se alza como la clave para comprender las elecciones de los jóvenes. En Chile, en el año 2012, según el Servicio de Información de Educación Superior (SIES), se matricularon en establecimientos de educación superior 351.426 alumnos y alumnas, un $51,8 \%$ corresponde a mujeres y un $48,2 \%$ a hombres (SIES, 2012). A pesar que existe una mínima diferencia en puntos porcentuales, se evidencia una leve feminización de la educación postsecundaria.

En relación a las áreas de conocimiento, de las carreras que estudian hombres y mujeres, existen diferencias importantes. Las mujeres tienden a estudiar carreras ligadas a la salud y la educación en mucha mayor proporción que los hombres; mientras que estos últimos triplican a las mujeres en el área de tecnología (SIES, 2012). A nivel de comparación con otros países de la región, Sikora y Pokropek sostienen: "En el Brasil y el Uruguay y la Argentina, las mujeres prefieren más las carreras científicas que los varones, mientras que en Chile y México es al revés" (Sikora y Pokropek, 2011, en CEPAL 2014, p. 26)

Ahora bien, la feminización de la educación superior en nuestro país no quiere decir que las mujeres acceden a mejores ingresos respecto de los hombres. En el sistema de retribución del mercado laboral aún se observan desigualdades de género (CEPAL, 2014). La evidencia más clara, es la brecha salarial existente entre la fuerza de trabajo femenina y masculina.

Al comparar la participación de hombres y mujeres en el mercado de trabajo chileno, se evidencia una participación mucho mayor de la población masculina. Para el año 2011, la Tasa de Participación de la Población Urbana en la Actividad económica en hombres era de $70,2 \%$, mientras que la de mujeres llegaba sólo a un 45,2\%. (CEPAL, 2011). 
En Chile, las mujeres representan más de un tercio de la fuerza laboral $(43,5 \%)$ y se concentran principalmente en los sectores de servicios sociales y de salud $(66,5 \%)$ y enseñanza (55,5\%) (Ver gráfico 1$)$.

\section{Gráfico 1}

Chile 2007: Distribución porcentual de los puestos de trabajo por sexo, según categoría económica (\% sobre el total)

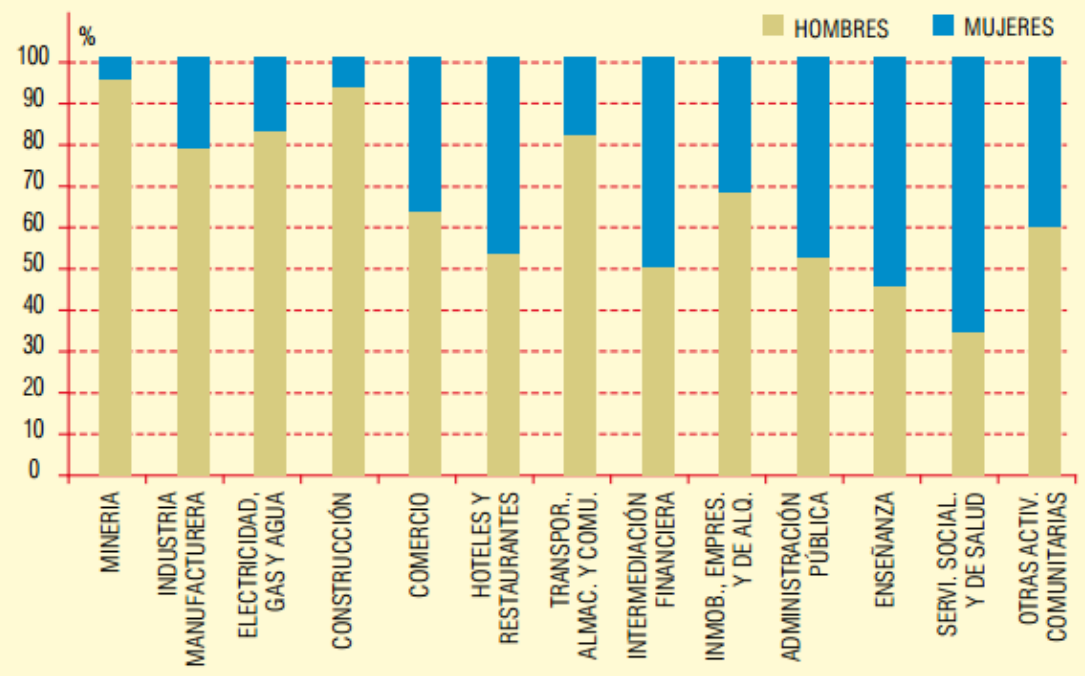

Fuente: Encuesta estructural de remuneraciones, costo mano de obra y empleo 2007

Fuente: Instituto Nacional de Estadísticas (INE, 2007). Encuesta Estructural de Remuneraciones, Costo de Mano de Obra y de Empleo: Boletín Informativo, Santiago de Chile.

En general, la población femenina en Chile se desempeña en empleos que implican remuneraciones más bajas (SERNAM, s.f.). De hecho, en el año 2009, los hombres ganaban en promedio por hora, remuneraciones $15,8 \%$ mayores que las mujeres Cabe destacar, que a medida que aumenta el nivel educacional, las brechas entre hombres y mujeres aumenta (ver tabla 1). 
Tabla 1

Chile 2009: Proporción relativa del ingreso y brecha salarial por hora promedio de la ocupación principal de las mujeres asalariadas respecto de los hombres asalariados, según nivel educacional

\begin{tabular}{|l|c|c|}
\hline \multicolumn{1}{|c|}{ NIVEL EDUCACIONAL } & PROPORCIÓN RELATIVA & BRECHA SALARIAL \\
\hline Educación Básica o Primaria & $86,1 \%$ & $-13,9 \%$ \\
\hline Humanidades o Educación Media Científico-Humanista & $79,5 \%$ & $-20,5 \%$ \\
\hline Educación Media Técnica Profesional & $83,5 \%$ & $-16,5 \%$ \\
\hline Centro de Formación Técnica incompleta (sin título) & $67,3 \%$ & $-32,7 \%$ \\
\hline Centro de Formación Técnica completa (con título) & $74,2 \%$ & $-25,8 \%$ \\
\hline Instituto Profesional incompleto (sin título) & $72,6 \%$ & $-27,4 \%$ \\
\hline Instituto Profesional completo (con título) & $71,3 \%$ & $-28,7 \%$ \\
\hline Educación Universitaria incompleta (sin título) & $77,5 \%$ & $-22,5 \%$ \\
\hline Educación Universitaria completa (con título) & $64,6 \%$ & $-35,4 \%$ \\
\hline Universitaria de Postgrado & $62,6 \%$ & $-37,4 \%$ \\
\hline
\end{tabular}

Fuente: Dirección del Trabajo (2011) El derecho a ganar lo mismo. Ley 20.348: Igualdad de remuneraciones entre hombres y mujeres, Temas Laborales N27, Dirección del Trabajo, Santiago de Chile.

Situación similar ocurre con la brecha de ingresos en relación al nivel socioeconómico. Mientras mayor es el nivel socioeconómico, la diferencia entre remuneraciones de hombres y mujeres se agudiza aún más. (Dirección del Trabajo, 2011)

De acuerdo a lo anterior, la situación laboral de las mujeres en Chile es más precaria que la de los hombres. Se evidencian "proporciones más altas de mujeres trabajadoras, en relación a los hombres, en los tramos de menores remuneraciones y con participaciones menores que ellos en los tramos de mayores remuneraciones, lo cual da cuenta de una persistente inequidad de género." (Dirección del Trabajo, 2011, p. 154)

Es importante observar esta realidad y sus posibles efectos bajo la mirada de Marta Lamas, quien menciona que el género se inscribe en la objetividad de las estructuras sociales y en la subjetividad de las estructuras mentales (Lamas, 2000). Estos antecedentes entonces, evidencian diferencias objetivas de género tanto en el ámbito de la educación como en el del trabajo y considerando, que el interés del artículo es dar cuenta de diferencias subjetivas de género en las relaciones existentes en el campo escolar, se intentará además revisar brevemente el rol del espacio escolar según los aportes de Bourdieu quien, nos señala que la relación de dominación de hombres hacia mujeres actúa en todos los espacios sociales: 
Una aprehensión realmente relacional de la relación de dominación entre los hombres y las mujeres tal como se estableció en el conjunto de los espacios y subespacios sociales, es decir, no únicamente en la familia sino también en el universo escolar y el mundo del trabajo (...). (1996, p. 126)

La escuela, entonces, es un espacio en el que se aprende a ser hombre y se aprende a ser mujer, en donde además, se aprenden los comportamientos adecuados a cada género. Las diferencias de género en el espacio educacional son concebidas entonces como naturales, verdaderas y racionales (Flores, 2005).

\section{Marco teórico}

\subsection{Del género y su identidad social virtual}

Cabe mencionar que entenderemos al género como la normatividad que impone dos sexos dicotómicos a los hombres y a las mujeres desde una matriz completamente heterosexual.

En nuestra sociedad, se tienen pautas sobre lo que significa e implica ser hombre o mujer. Se han naturalizado, y en consecuencia legitimado, diferencias que son producto de arbitrariedades culturales. Se "legitima una relación de dominación inscribiéndola en una naturaleza biológica que es en sí misma una construcción social naturalizada" (Bourdieu, 2000, p. 37). A esta relación de dominación, se le denomina violencia simbólica: poder que impone significaciones (en este caso, dominación de parte del hombre para con la mujer) establecidas como legítimas. De este modo, existe una manera determinada de ser hombre o mujer, que genera expectativas del comportamiento. (Reveco, 2011)

En otro sentido y como explica Goffman (1963), el medio funda elementos para categorizar a las personas junto a atributos en cada una de esas categorías. Así tratamos con "otros ya previstos", asumiendo de antemano en qué categorías se encuentran y cuáles son sus atributos. Estas anticipaciones las realizamos casi en forma inconsciente al relacionarnos con los demás y las convertimos en expectaciones, normativas y demandas, son finalmente imputaciones en propiedad, a esto se le denomina "identidad social virtual". Sin embargo los otros, poseen atributos o categorías propias y es lo que Goffman denomina "identidad social real".

En el caso de la identidad social virtual de género se ha establecido una dicotomía entre el hombre y la mujer, lo masculino y lo femenino en oposición a la identidad social real 
de género. La identidad social corresponde al imaginario sobre el género, mientras la identidad social real de género existe y puede ser demostrada en acciones (Goffman, 2006).

De acuerdo a la identidad social virtual de género se puede establecer:

- Existen dos géneros dicotómicos y excluyentes

- Que estos géneros, masculino y femenino son atribuibles a diferencia sexuales anatómicas y naturales

- Que la diferencia de género obedece a una diferencia sexual y que el género femenino se encuentra subordinado al masculino

- Que la identidad de una persona está definida por su identidad sexual y de género. (Lamas, 2000)

Entonces, la acción de categorizar a las personas, según Goffman (2006), de acuerdo a una serie de atributos, se realiza en base a juicios sociales y una de las tantas maneras de clasificar a las personas es según la variable sexo, entonces ¿Qué es ser hombre y qué es ser mujer en la sociedad actual?, las respuestas a dichas interrogantes se encuentra en pautas de comportamientos y atributos sociales legitimados asociados al género, expectativas normativas sobre cómo debe ser el hombre y cómo debe ser la mujer. En el caso de las mujeres, por ejemplo, se espera que sean "femeninas, es decir, sonrientes, simpáticas, atentas, sumisas, discretas, contenidas, por no decir difuminadas. Y la supuesta feminidad sólo es a menudo una forma de complacencia respecto de las expectativas masculinas (...)" (Bourdieu, 2000, p. 86)

Interesante es observar que cuando se produce una discrepancia entre la identidad social virtual y la identidad social real, se produce el estigma, entendiéndole a manera de una sanción generada por el alejamiento de lo que se concibe como identidad femenina o masculina, una diferencia no deseable. Esta estigmatización termina por marcar y disminuir a quienes buscan fracturar las diferencias establecidas.

Ahora bien, se señaló previamente que en nuestra sociedad impera la dominación masculina y que Bourdieu entiende esta dominación como la de "mayor eficacia simbólica" y "la transformación de la historia en naturaleza y de la arbitrariedad en natural". (Bourdieu, 2000). Dicha dominación hace uso de las instituciones de la educación para conservar el orden social así en la escuela, se enseña lo que es legítimo aprender. Lo que es legítimo aprender, en una cultura que así lo valida, producto de cultura de las clases y los grupos dominantes. 
Dentro de la dinámica intra-aula de los establecimientos escolares emerge lo que se podría evidenciar como las diferencias entre lo definido por currículo oculto y explícito. El primero de ellos, lo conforman los mensajes que se enseñan y aprenden en la escuela sin necesariamente estar consciente de aquello. De acuerdo a Flores, "el currículo oculto está formado, entre otros elementos, por creencias, por mitos, por principios, por normas y por rituales, que, de manera directa o indirecta, establecen modalidades de relación y de comportamiento de acuerdo con una escala de valores determinada". (Flores, 2005, p. 15). El segundo, corresponde al programa de estudios que se encuentra declarado explícitamente por la institución educacional. El contraste entre ambos currículos es de suma importancia toda vez que enuncian realidades certeras del aula.

\section{Abordaje Metodológico}

La presente investigación fue de carácter cualitativo y correspondió a un estudio de caso que pretendió realizar un proceso de comprensión. "La investigación cualitativa describe el orden de significación, la perspectiva y la visión del investigado" (Canales, 2006, p. 20). Se ha buscado conocer los significados que los sujetos participantes de la acción educativa, atribuyen a dichas interacciones dentro del aula. Debido a lo anterior, es necesario profundizar en las relaciones sociales establecidas entre estudiantes y docentes.

El estudio tiene una orientación descriptiva-interpretativa. Descriptiva, porque el escenario de las interacciones de ambos actores es la situación de aula, por lo que se describen dos procesos que ocurren al interior de ésta, en otro sentido, interpretativa, dado que las situaciones de aula fueron analizadas y comprendidas, mediante las referencias teóricas expuestas en el apartado anterior. En relación a la muestra, los sujetos participantes de la investigación se clasifican en dos categorías.

Alumnos: Entendido como hombres y mujeres de segundos medios de dos comunidades de enseñanza particular pagada científico humanista, orientadas a la inserción del alumnado a la educación superior universitaria diurna. Se estableció como requisito adicional, que estuvieran cursando las asignaturas de física, matemática y química en el plan común del currículo, además de los electivos. Participaron 93 alumnos y alumnas, observados. 45 mujeres, 48 varones. Entrevistas en profundidad 9: 4 varones, 5 mujeres. 2 Grupo Focales.

Docentes: Entendido como hombres y mujeres que se hubiesen desempeñado en el cargo de profesores de las especializaciones estudiadas en los segundos medios, por lo 
menos dos años con los mismos estudiantes. Se favoreció este criterio de selección para que el estudiantado realizara una evaluación fundada en una vinculación con ellos, comunicación y conocimiento, más allá de una experiencia fugaz. Total 12: 5 mujeres, 7 varones. 1 Grupo focal 8 participantes. 4 profesoras, 4 profesores.

\subsection{Técnicas e instrumentos}

Se utilizaron tres técnicas de recolección de información: Entrevistas, Observaciones de aula (no participantes) y grupos focales (ver figura 1):

Figura 1

Técnicas de recolección de información utilizadas

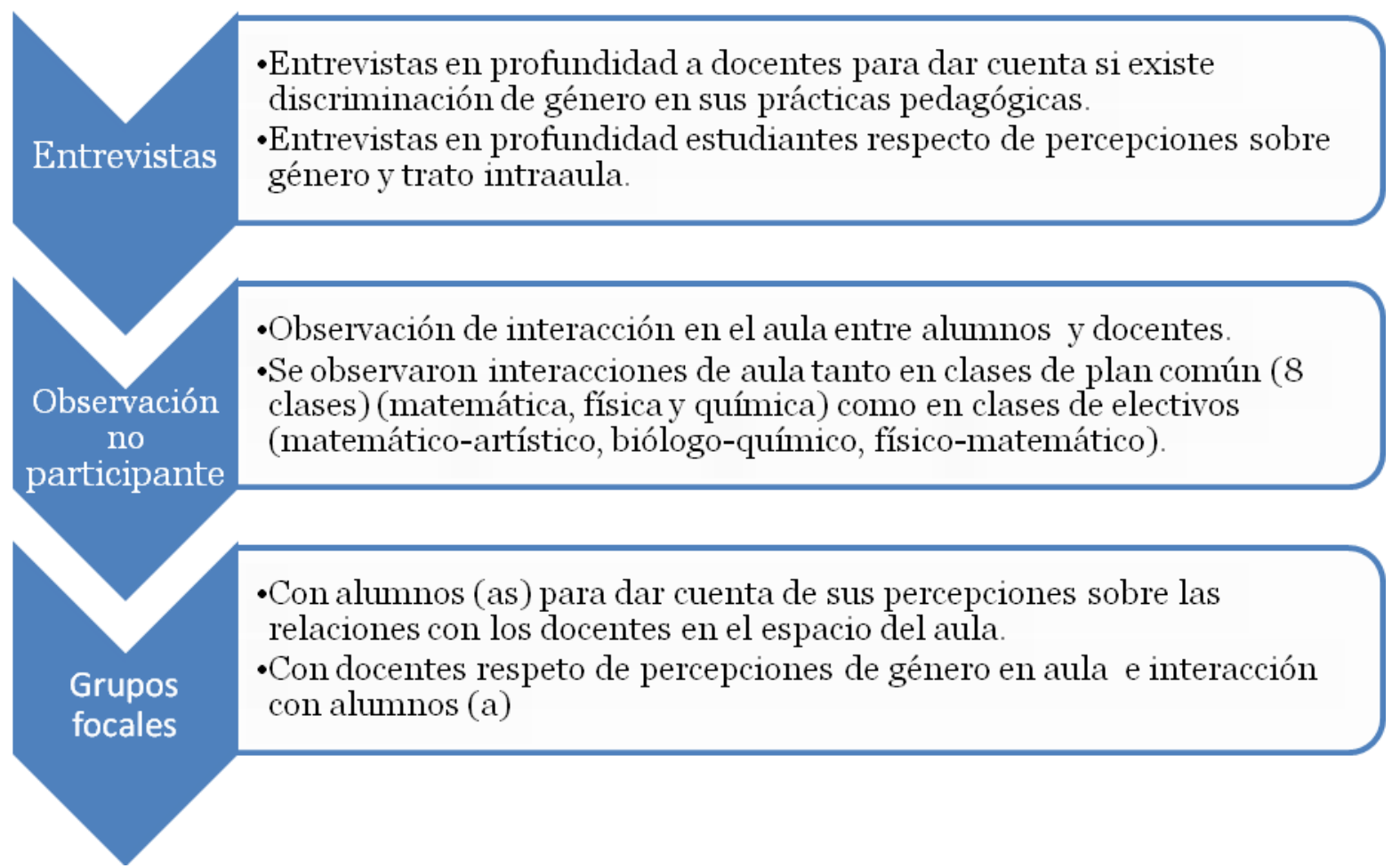

Fuente: elaboración propia.

\section{Plan de análisis}

Para realizar el análisis de contenido, la información recogida se debió convertir en algo susceptible de describir y analizar. Es por esta razón, que en primer término, se identifican las temáticas emergentes en el discurso de los sujetos estudiados surgiendo tres grandes temas (ver tabla 2): 
Tabla 2

Categorías de análisis

Categorías de análisis

Creencia sobre la

igualdad o desigualdad

de género.

Interacción intra aula,

cuerpo

docente/alumnado.

Legitimización de la creencia de la igualdad o desigualdad de género.

\section{Defínición}

Esta categoría hace referencia a lo que Bourdieu llama las estructuras cognitivas de la sociedad, donde impera la dominación masculina (Bourdieu, 2000). A esta dimensión se suma lo que el mismo autor hace referencia con el concepto de los esquemas inconscientes de percepción y de apreciación, es decir, no solamente los estereotipos de género a nivel consciente, sino completamente interiorizados en el inconsciente.

En las situaciones de aula, tanto los alumnos y, quizá, en mayor grado, las alumnas responden a las expectativas de la autoridad institucional del cuerpo docente tratando de no divergir del estereotipo e, incluso, en algunos casos, utilizando, consciente o inconscientemente, el estereotipo a su favor.

Intenta rescatar el significado que el cuerpo docente y el alumnado, varones y mujeres, otorgan a la igualdad 0 desigualdad entre hombres y mujeres. Socialmente, el sistema de sexo-género es binario y rige, en este sentido, la dicotomía de masculino o femenino, tomando a este "o" como una disyunción

Fuente: elaboración propia

Una vez identificados los códigos principales, se realizó un análisis del discurso recogido en entrevistas y grupos focales, tomando como criterio el Análisis Estructural de $A$. J. Greimas (1976).

Greimas (1973) propone una estructura elemental de la significación a partir de un esquema de oposiciones semánticas. Los semas se relacionan y ordenan jerárquicamente. Este tipo de análisis permite construir un modelo que se divide en tres fases: (a) Recomposición de la estructura o identificación de las realidades o temas (b) dinamización de la estructura o distribución de las oposiciones y asociaciones identificadas (c) interpretación global o reconstrucción de los modelos simbólicos subyacentes a los textos.

Este análisis involucra las siguientes tareas: primero se describe el discurso en función de los códigos que significan la unidad mínima de sentido común -como el signo en la lingüística estructural- basado en el principio de disyunción y conjunción. Por ejemplo, a un determinado A "Masculinos", se le va a oponer un determinado B "Femeninos".

A estas oposiciones se le asigna un eje semántico que es denominado totalidad y al cual pertenece la oposición construida. De esta manera, que el código se constituye simultáneamente por el principio de oposición (disyunción) y el de conjunción (totalidad común a los términos oposicionales). Si en el texto no aparece explicitada la oposición, se construye la misma como su opuesto lógico que aparecerá entre paréntesis. 
Lo anterior se puede sintetizar en la siguiente fórmula:

$$
(A / B=T)
$$

La segunda tarea consiste en la definición de estructuras de relaciones que existen entre los códigos. Están ordenadas y este orden del texto constituye la estructura del sistema. Las estructuras poseen calificación positiva (+) o negativa (-), calificación que está denotada en el texto.

El Análisis Estructural da lugar a tres tipos de estructura:

- Estructura paralela: compuesta de dos o más disyunciones reunidas por las relaciones de mutua implicación. Expresan una distinción clara entre los términos que forman los códigos (ver figura 2).

Figura 2

Ejemplo Análisis Estructural A

\begin{tabular}{lllll}
\hline A & & \multicolumn{1}{c}{ B } & \\
Pertenece & $/$ & Excluido & Grupo Social \\
Poseer & $/$ & Desposeer & Territorio \\
Cultura y Tradición & $/$ & Sin cultura ni tradición $=$ & Ciudadanía \\
Pertenecer, poseer, cultura & Excluido, desposeer, sin cultura ni tradición \\
y tradición & & & \\
\hline
\end{tabular}

Fuente: Andrade y Miranda, 2001.

- Estructura jerarquizada: los términos que forman los códigos tienen sub-códigos, que entregan más especificaciones a la clasificación. Esta estructura se forma cuando uno de los códigos cumple dos funciones: es un subcódigo y es la totalidad de un nuevo código (ver figura 3).

\section{Figura 3}

Ejemplo Análisis Estructural B

\begin{tabular}{|c|c|c|}
\hline \multicolumn{3}{|c|}{ T1 =Alumnos que terminan la enseñanza básica } \\
\hline$(+)$ & l & $(-)$ \\
\hline A1 & I & $\mathrm{B} 1=\mathrm{T} 2$ \\
\hline El $50 \%$ va a la media & & (desertan) \\
\hline-+ & I & - - \\
\hline A2 & I & B2 \\
\hline Trabaja & / & queda esperando bebe \\
\hline
\end{tabular}

Fuente: elaboración propia 
- Estructura cruzada: da cuenta de una forma de organización entre dos disyunciones donde los términos no se implican el uno al otro. Permite construir cuatro posibilidades de combinación de los términos de las dos disyunciones (ver figura 4).

Figura 4

Ejemplo Análisis Estructural C

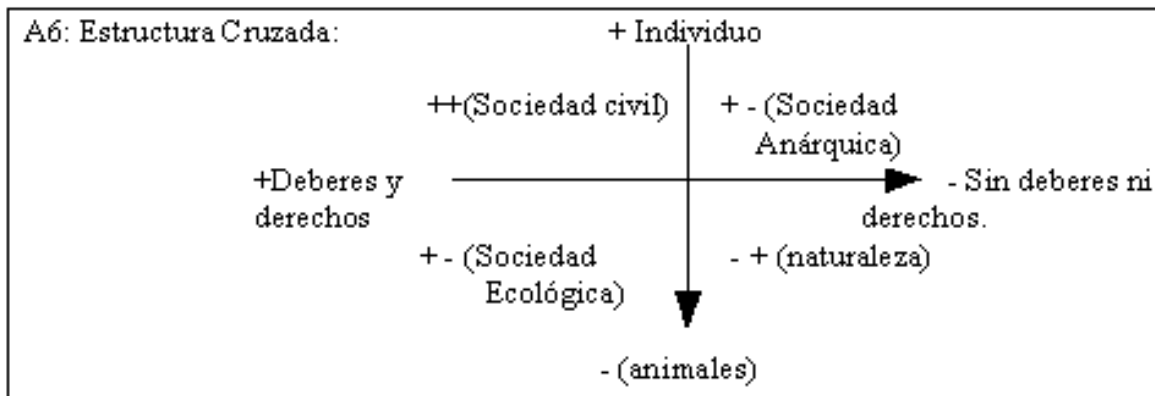

Fuente: Andrade y Miranda, (2001).

Una vez identificadas las estructuras del discurso de alumnos y docentes, se procede a la interpretación de acuerdo a las formulaciones del marco teórico seleccionado.

\section{Resultados}

Los resultados de la investigación se presentan de forma separada para docentes y alumnado. En el apartado de conclusiones se hará un análisis integrativo de ambas miradas.

\subsection{El punto de vista de los docentes}

Para los docentes la desigualdad de género queda legitimada por la naturaleza y va a determinar las capacidades cognitivas según sexo: Mientras los hombres poseen mayores habilidades cognitivas para las ciencias, las mujeres poseen menores (ver figura 5).

Figura 5

Estructura Jerarquizada legitimación desigualdad de género por parte de docentes hombres

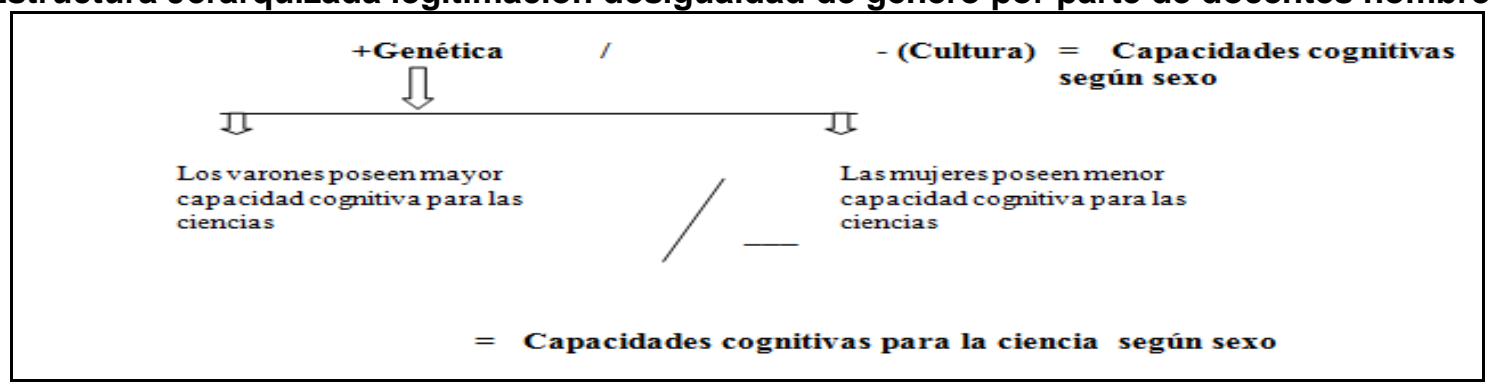

Fuente: elaboración propia. 
Partiendo de la convicción de que la igualdad entre los hombres y las mujeres no existen, y que la inferioridad de la mujer se debe a la genética o la naturaleza o la herencia, no resulta sorprende que esta misma naturaleza explique la desigualdad en capacidad de aprendizaje científico por parte de las alumnas. Lejos de una autocrítica que los llevaría a reformularse sus prejuicios y, por lo tanto, a no reproducir las desigualdades y estigmatizar al o la diferente, los profesores se asientan en la naturalización de las diferencias de género (ver figura 6).

Figura 6

Legitimación de la desigualdad entre los sexos y la diferente inserción en el mundo profesional adulto de estudiantes según el profesorado

\begin{tabular}{|l|l|l|}
\hline + Genética $/$-(Cultura) & $\begin{array}{l}\text { Legitimación de la } \\
\text { desigualdad entre los } \\
\text { sexos }\end{array}$ \\
$\begin{array}{l}\text { Ciencias, Ingenierías, } \\
\text { Técnicas }\end{array}$ & $\begin{array}{c}\text { Mujeres: } \\
\text { Humanidades, Servicios }\end{array}$ \\
& $=$ Inserción en elmundo profesional adulto
\end{tabular}

Fuente: elaboración propia.

De esta manera, para los docentes, hombres y mujeres poseen diferentes atributos y éstos los constituyen como personas. Esto tiene un efecto crítico en la inserción de ambos en la vida adulta, etapa de la vida donde se invierte el status privilegiado de los varones (ver figura 7).

Figura 7

Legitimación de la desigualdad de género y la diferente inserción de estudiantes en el mundo afectivo adulto

\begin{tabular}{|c|c|c|}
\hline+ & $/$ - (Cultura) & $\begin{aligned} & \text { Legitimación de la } \\
= & \text { desigualdad de género }\end{aligned}$ \\
\hline UL & & य \\
\hline $\begin{array}{l}\text { Inserción en el mundo } \\
\text { adulto afectivo: desde la } \\
\text { maternidad, las mujeres son } \\
\text { cálidas, emocionales, } \\
\text { preparadas para el cuidado } \\
\text { y el amor al otro }\end{array}$ & & $\begin{array}{l}\text { Inserción en el mundo adulto } \\
\text { afectivo es deficiente en el caso de } \\
\text { los varones: incapaces de cuidar } \\
\text { no sirven como padres afectivos, } \\
\text { fríos, incapacitados } \\
\text { emocionalmente. }\end{array}$ \\
\hline
\end{tabular}

Fuente: elaboración propia 
En el caso de la inserción en la vida secundaria de hombres y mujeres, nos encontramos con la presencia de los mismos estereotipos a favor de la mujer (ver tabla 3 ).

Tabla 3

Levantamiento de Atributos Percepciones Docentes

Alumnos

Atributos personales según profesores de:

万)

"el hombre por su incapacidad maternal es menos de sentimientos"

- -(los hombres son menos maduros, comprenden menos)

- (los hombres desordenan las clases)

- (biológicamente el hombre es menos maduro que la mujer)

- (el hombre carece de la capacidad de ser buen padre)

- -(el hombre es más abstracto es más frío, calculista que ellas)

- -(el hombre es más bruto) "la mujer por su capacidad maternal es más de sentimientos"

"hay más madurez en las mujeres, son capaces de comprender más"

"se nota diferencia si hay más mujeres, las clases son más ordenadas"

"Yo creo que biológicamente la mujer madura antes que el hombre"

"El hecho de tener la capacidad de traer vidas al mundo las prepara, el hombre es más liviano, "chacotero" . En una pareja por ejemplo, al enterarse de la noticia de una nueva vida en camino, la mujer lo acepta con mayor rapidez que el hombre"

"Esto es genético la mujer tiene capacidad sentimental, está más capacitada para el humanismo, el cuidado, la maternidad"

"A las mujeres se les trata distinto como ya dije no son iguales, son más delicadas"

Fuente: elaboración propia

En términos de atributos personales y humanos se invierte la significación dada a la desigualdad de género en términos de capacidades de aprendizaje científico y cognitivo general. El cuadro humano que los profesores hombres ofrecen de los alumnos varones, en otro sentido, también de sí mismos, es el de una criatura inmadura, incapacitada de otorgar cuidados, con una más que deficiente capacidad paternal, con menos capacidad sentimental, más frío o menos cálido, comprenden menos en términos de lo que hoy se denomina la inteligencia emocional, e incluso más calculador, menos auténtico. Así, el 
estereotipo del varón que es inculcado desde los prejuicios de los profesores de su mismo sexo es aprendido por el alumno y, no sorprendentemente, reproducido.

En síntesis, ninguno de los docentes de la muestra cree en la igualdad de hombres y mujeres, todos coinciden en que la capacidad de aprendizaje científico es mayor en el caso de los alumnos. La explicación de esta diferencia no deja lugar a ninguna alternativa en tanto se ha naturalizado esta diferencia y, como menciona uno de los profesores, incluso las ciencias han legitimado esta diferencia. Esto, en la mayoría, se traduce en un trato distinto.

Finalmente, se intenta un análisis que revele cuál es la realidad de género que predomina en el cuerpo docente en los segundos medios estudiados y cuál es aquella realidad 0 realidades excluidas (ver figura 8 ).

Figura 8

Realidad de género en el cuerpo docente

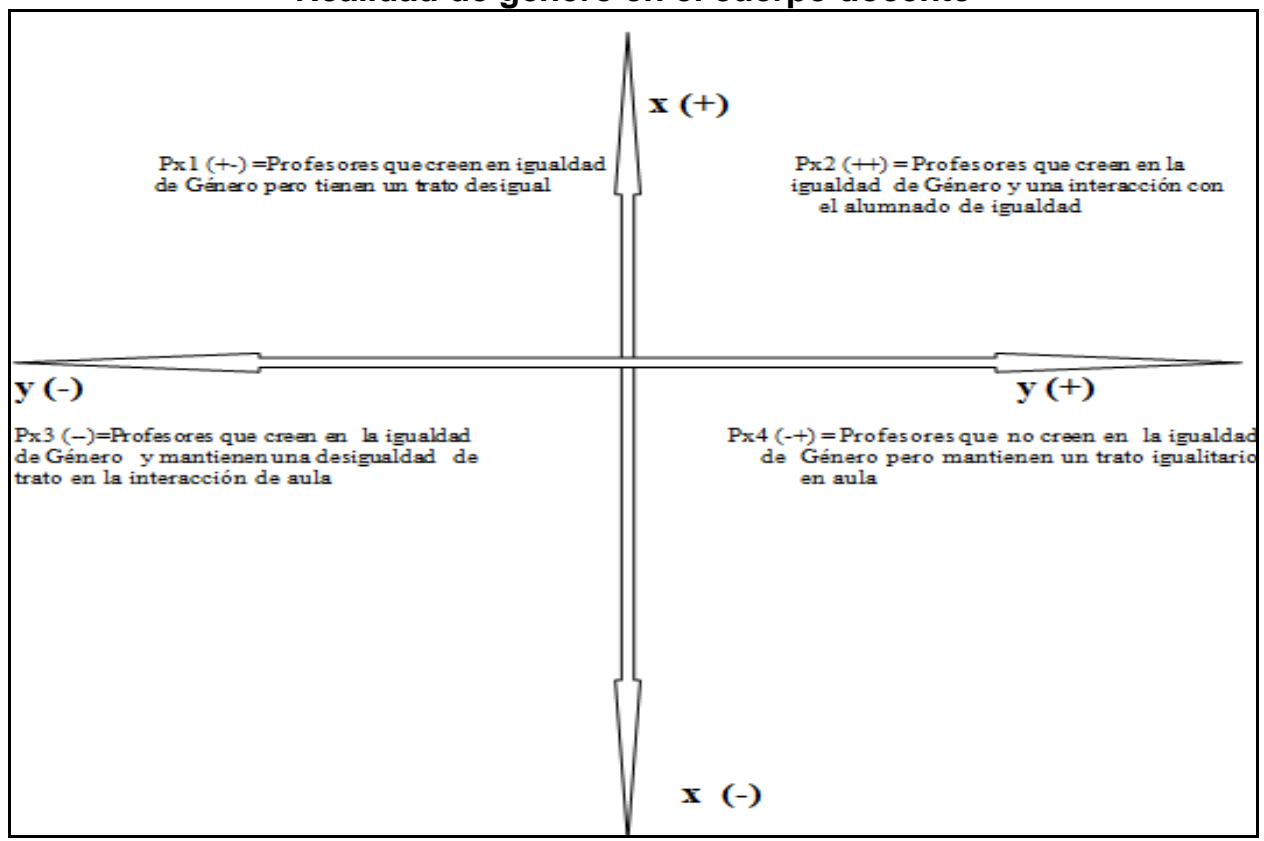

Fuente: elaboración propia

Así, la mayoría de los profesores se sitúan en la realidad Px3 mientras que la realdad Px2 desgraciadamente se construye como la realidad excluida. No existen en la observación realizada profesores que crean en la igualdad de género y que operacionalicen esta convicción en un trato igualitario en las clases de ciencia. Por otra parte, existen docentes que consideran que la desigualdad de género no implica un trato desigualitario en el aula, situándose así dentro de la realidad Px4, intentan ser equitativos en aula, más subyace en 
ellos enfoques machistas. Finalmente, los profesores que creen en la igualdad de género, pero no la practican en el aula, es porque reconocen la responsabilidad de la educación y de la sociedad así como su participación activa en reproducir la desigualdad en el "mundo machista".

Si deseamos profundizar en la categoría de creencia de la Igualdad o desigualdad de género, podemos mencionar que aquí emerge lo que Bourdieu menciona como dominación masculina (Bourdieu, 2000). Más importante aún, lo que los Estudios de Género llaman el inconsciente masculino. Mulvey (1975), Kaplan (1983), y Kuhn (1982) en su análisis de la mirada masculina, como la norma de la mirada en la sociedad patriarcal, ellos estudian a través del análisis cinematográfico, este inconsciente colectivo y sus dificultades para lidiar con la imagen de la mujer, concluyendo que ésta le es insoportable en términos psicológicos (complejo de castración) compensándolo con otros mecanismos psicológicos como el voyeurismo y el fetichismo. De igual manera, en los estudios críticos literarios feministas, se plantea la imposibilidad por parte del canon masculino imperante, de concebir una escritura femenina que, primero, se alza como una imposibilidad sobreviviendo la significación de la mujer a nivel oral, y, luego, como una diferencia, una inflexión irremediable. Desde el psicoanálisis propiamente dicho y desde la lingüística Irigaray (1985) y Violi (1996) plantean la imposibilidad de lo femenino. La mujer, para la primera, es irrepresentable en el lenguaje androcéntrico; mientras que Violi explica cómo la lengua se levanta en el universal que no es sino un travestismo de lo masculino que se alza en trascendente-muy similarmente al planteo filosófico de Beauvoir. Si para Simon Beauvoir el Otro es femenino y subordinado, para Violi lo femenino emerge como lo singular. En antropología, ya Ortner y Whitehead (1991, primera edición en español) señalaba el funcionamiento del inconsciente masculino que dicotomiza masculino/femenino como equivalente a cultura/naturaleza. Como veremos, desafortunadamente, esto no es una invención o una pobreza de la teoría, todo lo contrario:

“. . es que yo creo que ya lo dijimos, que las mujeres son mucho más maternales” (Alumna Colegio Particular Pagado Laico)

Podemos entonces observar que la problemática de la reproducción de las identidades sociales virtuales de género a través de la educación requiere una revisión del cómo la práctica docente se transforma en una herramienta de reproducción social. Cabría considerar además que toda cultura es arbitraria y segundo, su significado es social, (Bourdieu y 
Passeron, 1996) mencionan que toda sociedad es una suma de relaciones de fuerza que se disimula a través de la violencia simbólica. Definen a esta como "todo poder que logra imponer significados y aplicarlos como legítimos disimulando las relaciones de fuerza en las que se basa su fuerza". La escuela y su acción pedagógica realizada por el cuerpo docente "es objetivamente una violencia simbólica en cuento impone a través de su poder, una arbitrariedad cultural". La socióloga Silva Donoso en su análisis de la educación: En Chile hasta comienzos de la década de 1990, la escuela continua reproduciendo las diferencias de géneros, tal y como lo hacen otras instituciones sociales. En el caso de los establecimientos educacionales es el currículo oculto es el que perpetúa y refuerza los estereotipos sexuales (Silva Donoso, 1989).

Desde un punto de vista teórico resulta significativo y revelador que el concepto de género en tanto construcción cultural y social forma parte de esta cultura legitima que para los sociólogos franceses enmascaran la cultura de las clases y los grupos sociales dominantes transformando la cultura socialmente definida como la cultura objetiva. $Y$ es objetiva en dos aspectos relevantes: resuelta indiscutible y rechaza la cultura de los otros grupos sociales.

\subsection{El punto de vista del alumnado: resistencia, inversión del orden y resignificación}

La problemática se complejiza cuando nos preguntamos sobre el punto de vista del estudiantado: ¿Aceptan esta desigualdad en la interacción cuerpo docente-alumnado o, por el contrario, ¿articulan respuestas diversas? Y, ¿cómo se da este trato igualitario en función de las interacciones inicialmente señaladas desde la percepción del alumnado?

En términos de la categoría de la creencia en la igualdad de género y la legitimación de la desigualdad entre hombres y mujeres, podemos expresar estas percepciones de acuerdo a las siguientes significaciones por parte de alumnado: deben aceptar ante la experiencia concreta, la situación de aula, la discriminación, pero la relación entre ellos intenta ser comunitaria e igualitaria.

Para la mayoría del alumnado impera o debiera imperar la igualdad entre los sexos, sin embargo, ante la experiencia concreta de la sociedad de la dominación masculina, la explicación de la desigualdad y de la subordinación femenina se explica, a diferencia de los profesores, no como parte de la naturaleza sino como un constructo socio-cultural. 
En lo que concierne a la categoría que trata sobre la interacción en el aula, y si nos concentramos en una interacción desigual manifiesta en el trato, entonces, queda claro que el alumnado coincide acerca del trato diferenciado por parte de los profesores, que se presenta como generalizado y claramente identificado por ellos. Así, desde el punto de vista del alumnado, en la experiencia cotidiana del aula la desigualdad en la interacción con el cuerpo docente en función del sexo es un problema que deben confrontar (ver figura 9).

Figura 9

Percepción de alumnos y alumnas sobre la desigualdad en la interacción con el cuerpo docente

$\mathbf{x}(+)$

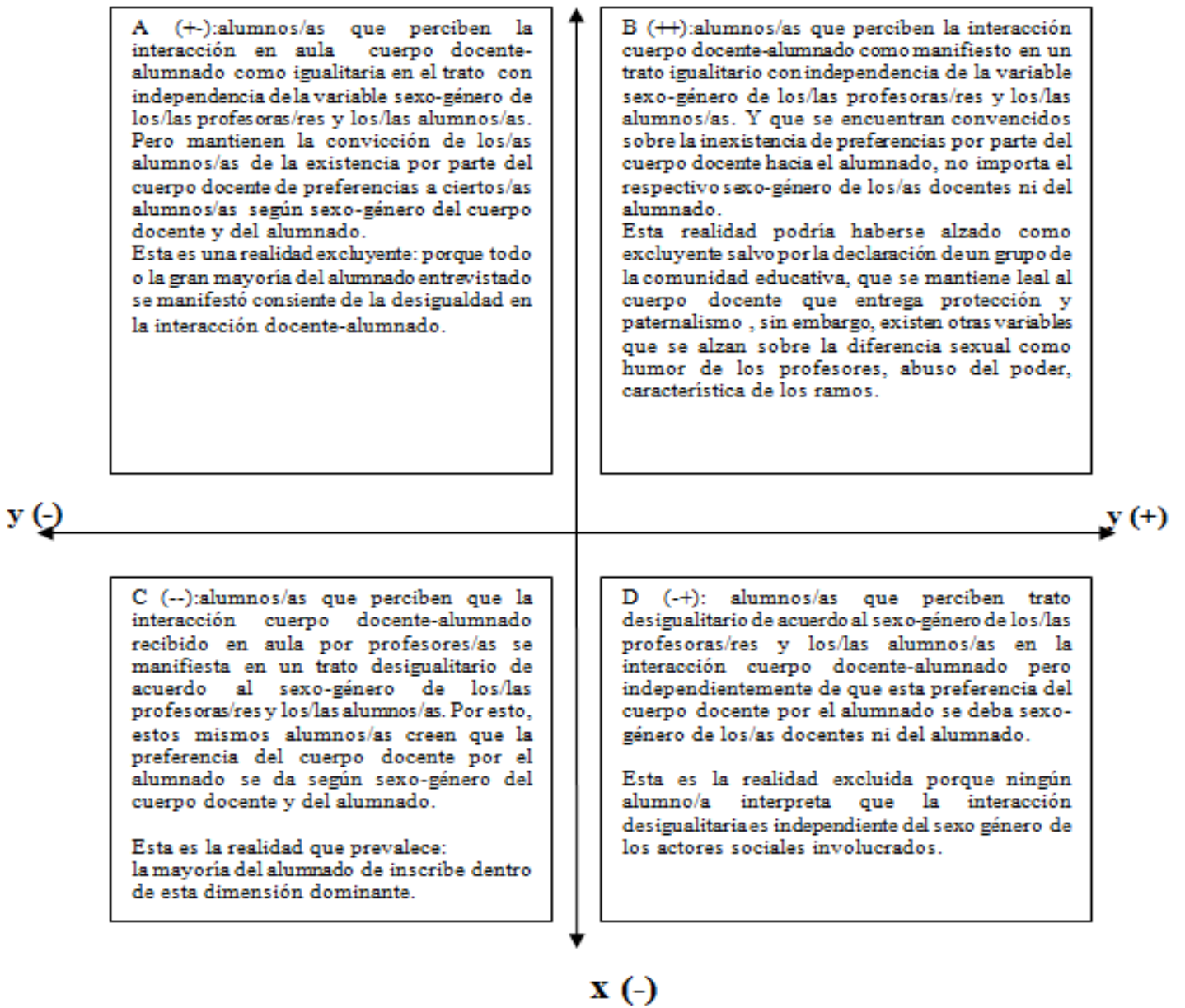

Fuente: elaboración propia

Donde Eje $\mathrm{x}=$ Percepción del alumnado sobre la interacción cuerpo docente-alumnado manifiesto en el trato recibido en aula por docentes en relación a su sexo-género.

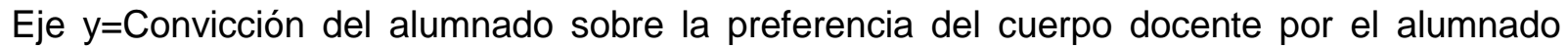
según sexo-género. 
Así,

X $(+)=$ Percepción de los estudiantes sobre la interacción cuerpo docente-alumnado recibido en aula por profesores manifiesto en el trato igualitario con independencia de la variable sexo-género de los profesores y el alumnado.

x (-)=Percepción de los estudiantes sobre la interacción cuerpo docente-alumnado recibido en aula por profesores, manifiesto en el trato desigualitario de acuerdo al sexo-género de profesoras y alumnos.

y $(+)=$ Convicción de los estudiantes sobre la inexistencia de preferencia del cuerpo docente por ellos, no importa el respectivo sexo-género de los docentes ni el de estudiantes.

Y (-)=Convicción de los estudiantes sobre la preferencia del cuerpo docente por el ellos según sexo-género de los docentes y del alumnado.

Finalmente, es necesario destacar que la categoría " $A$ " es una realidad excluyente, porque la mayoría del alumnado entrevistado se manifestó consciente de la desigualdad en la interacción docente-alumnado. La categoría "C" es la realidad que prevalece, ya que la mayoría del alumnado se inscribe en esta dimensión dominante. La categoría "D" corresponde a la realidad excluida, ya que ningún alumno o alumna interpreta que la interacción desigualitaria es independiente del género de los actores sociales involucrados.

Ahora bien, respecto de la legitimación de las diferencias de género por parte del alumnado, una alumna expresa: "Las mujeres somos más tranquilas". Si nos detenemos en esta afirmación, la traduciríamos de acuerdo al siguiente eje de significación (ver figura 10).

Figura 10

Formas de ser disciplinado según sexo

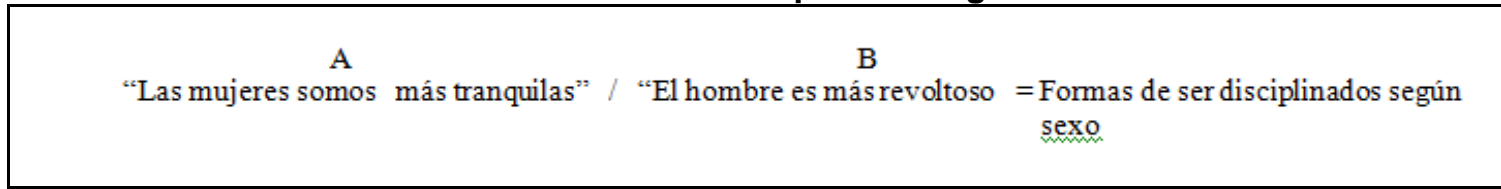

Fuente: elaboración propia

Al traducir el eje de significación anterior en términos de semas, se obtiene la siguiente fórmula (ver figura 11). 
Figura 11

Atributo de disciplina en la alumna

\begin{tabular}{ll|}
$\mathrm{S}$ (es la propiedad de) A \\
\hline $\mathrm{S}$ (disciplinada) A $=$ Alumna
\end{tabular}

Fuente: elaboración propia

De acuerdo a esta fórmula, el rasgo distintivo es el sema que permite identificar a la alumna como disciplinada significa que es una propiedad suya ser disciplinada en oposición al varón (V). El hombre se caracteriza por ser indisciplinado y alterar el orden en la situación de clase. De esta manera el ser más disciplinada se alza como una de las cualidades o elementos que constituyen al término objeto (A). Es así como en la situación de clase se impone el siguiente sistema sémico (ver figura 12).

Figura 12

Atributos personales según sexo

\begin{tabular}{ll|} 
"Tranquilas" vs "Revoltoso" \\
\hline Atributos personales según sexo
\end{tabular}

Fuente: elaboración propia

Donde la totalidad "formas de ser disciplinados según sexo" del inicial eje de significación, pasa a subordinarse al eje a "Atributos personales según sexo" de acuerdo a la siguiente estructura jerárquica (ver figura 13).

Figura 13

Formas de ser disciplinado según sexo

Hombres=Activos $/$ - (Mujeres=Pasivas) $=$ Atributos personales según sexo
Alumnas Disciplinadas $/$ - (Alumnos Indisciplinados) $=$ $\begin{gathered}\text { Formas de ser } \\ \text { disciplinado } s / \mathrm{sexo}\end{gathered}$

Fuente: elaboración propia

Frente a lo anterior, se sugiere la siguiente aproximación a explicación: cuando la alumna afirma que las mujeres son más tranquilas y que los profesores "sienten que deben 
tratar en forma más delicada a las mujeres" está realizando una manipulación de los estereotipos de género subvirtiéndolos a su favor e instaurando una resistencia. Las alumnas asumen una máscara de obediencia de acuerdo a la cual actúan de acuerdo a las anticipaciones expectativas de los profesores, reproduciendo la identidad social virtual de género. Las alumnas continúan actuando desde la máscara de la obediencia de ser "más tranquilas" que los varones en clase, acomodándose a las anticipaciones de la identidad social virtual de género de los profesores. Al reproducir el estereotipo de género (mujeres como pasivas) reciben un mejor trato personal en la interacción con el cuerpo docente.

Los anteriores procesos quedan enmarcados en la creencia de la desigualdad de género y su legitimación, en el marco íntimo, cara a cara y cotidiano de la situación de clase. Como Goffman (2006) indicaba esta situación microsociológica produce relaciones donde las anticipaciones y las respuestas a las mismas pasan a nivel inconsciente de tal manera que solamente se toma conciencia de la experiencia en sí cuando se violan las identidades sociales virtuales con las inevitables identidades sociales reales, en tal caso se produce una estigmatización. En las situaciones de aula, tanto los alumnos y, quizá, en mayor grado, las alumnas responden a las expectativas de la autoridad institucional del cuerpo docente tratando de no divergir del estereotipo e, incluso, en algunos casos, utilizando, consciente o inconscientemente, el estereotipo a su favor. Interesante resulta recordar que estas situaciones vividas por alumnos/as en sala, nos remite al acto no meditado, no mediado, a lo que Bourdieu (2000), tomando de la fenomenología de Husserl (1962) denominaba "la actitud natural". Esta "actitud natural" tal como la perciben los/as alumnos/as se manifiesta en que la mayoría de ellos percibe un trato desigual en las interacciones de aula. Los estudios de educación y género han tomado la situación de clase como una dimensión privilegiada en tanto se produce la interacción de forma espontánea siempre que se trate de intervenir como observador de la manera más controlada. Sin embargo, es tal la fuerza de la inercia de lo cotidiano, que luego de irrumpir en la clase, muy pronto la interacción vuelve a retomar su ritmo y modo. Como hemos visto, todos los docentes son partidarios, salvo uno profesor de un trato desigual con alumnos y alumnas. A su vez, los alumnos/as son conscientes de esto y critican por igual a quien promueva esta diferencia ya pertenezca al cuerpo docente o al alumnado:

si son diferentes entre hombres y mujeres, los profesores más que nada son los que marcan las diferencias en torno a los alumnos" (Alumno Colegio Particular Pagado Laico) 
"Ahí va con el tipo de enseñanza que acabo de decir 'denante' por ejemplo, un hombre cuando se equivoca en una prueba es no y no y chao no más, te conformai, pero con una mujer (en tono irónico) 'si, si puede ser, y un 0,5 más'o sea es eso lo que va creando [que] la mujer . . sea un poco más flexible y un hombre sea estricto y cierto que cambian cuando salen de la sala vuelven a ser civiles, vuelven a ser lo que son pero, la manera de sensibilidad entre uno y otro se mantiene" (Alumno Colegio Particular Pagado Laico)

Así la transmisión de los denominados bienes simbólicos, tienen en este espacio estudiado, un magno ejemplo. Es interesante recordar que ya Bourdieu, en el 2000, se centraba en el estudio de las estructuras e inconscientes sexuales "de una sociedad androcéntrica". (Bourdieu, 2000) Sin embargo, como explica la académica italiana Teresa de Lauretis, los Estudios de Género se caracterizan por analizar en su conjunto distintas diferencias como las de género, clase social, étnicas, atareas, orientación sexual, entre otras. (De Barbieri, 1993) De hecho, para esta autora, esto es lo que caracteriza a la teoría y a la metodología de los Estudios de Género.

De Lauretis planteó que esta perspectiva entregó un nuevo horizonte de conocimiento, esto es, un horizonte de significados que han sido reformulados planteando nuevos conceptos y estrategias retóricas es decir, "otro tipo de discursos", nuevos modos de interpretación, de realizar inferencias, de revelar premisas implícitas. Entre estos conceptos surge una nueva comprensión de la identidad como: "múltiple, cambiante y frecuentemente contradictoria. . una identidad hecha con base en representaciones heterogénicas y heterónoma del género, la raza y la clase, y ciertamente construida a menudo a través del lenguaje y culturas diversos" (De Lauretis, 1991, p. 73).

Con todo lo anterior podemos entonces pensar que no es casual que el mismo Bourdieu califique a la dominación masculina como la de "mayor eficacia simbólica": "la transformación de la historia en naturaleza y de la arbitrariedad en natural”. (Bourdieu, 2000, p. 12) él se expresa, en términos de "dominación masculina" en tanto se refiere a la diferencia entre lo masculino y lo femenino en "su carácter arbitrario y contingente, y por otra simultáneamente sus necesidad socio-lógica". De esta manera en su trabajo de 1998 se propone como objeto de estudio el análisis de la dimensión simbólica de la dominación masculina en la que ve el mejor ejemplo de la imposición del "orden establecido con sus relaciones de dominación sus derechos y sus atropellos, sus privilegios y sus injusticias" 
(Bourdieu, 2000, p. 11). La estudiosa Sara Kries, por su parte, en sus observaciones al sistema de la enseñanza chilena en función de su experiencia en la educación informal y su planteo por una educación emancipadora afirma que este proyecto político de transformación “implica entender la realidad como una totalidad compleja, dinámica e histórica y, por lo tanto, cuestionadora de las explicaciones de "simples de lo 'natural'”. (Kries, 1995, p. 87)

\section{Conclusiones}

En términos generales, se ha corroborado la existencia de discriminación de género por parte del grupo docente hacia el alumnado con el imperio de identidades sociales virtuales que se imponen a las reales. Este actúa en función de comportamientos y "esencias" femeninas y masculinas que operan en tanto anticipadas y esperadas. En cambio, el alumnado otorga significados diferentes respecto a la discriminación de género. Aquí, los significados otorgados a la diferencia de género resultan altamente disímiles porque la gran mayoría del alumnado expresa su creencia en la igualdad entre hombres y mujeres. Sin embargo, sus creencias diferían con la interacción que tenían con los docentes en el aula. Manifiestan que se notan las diferencias de trato por parte de los docentes según el género del alumnado y lo explican en función de comportamientos machistas entendidas como relaciones (inter)personales cotidianas.

Los resultados muestran que la totalidad de la población docente con la que se trabajó, establece una relación de enseñanza-aprendizaje en aula congruente con las identidades virtuales de género. Así, todos se expresaron en contra de la capacidad de las mujeres para el aprendizaje científico explicando esta afirmación en función de la natural capacidad emocional femenina lo que, consecuentemente, las inhabilita para un saber que presentan como frío y objetivo. Así, observamos lo que teóricos como Bourdieu, Butler, y de Lauretis han denominado la naturalización de una diferencia y una jerarquía cultural. (Bourdieu, 2000; Butler, 1990, 1977; de Lauretis, 1991, 1993)

El profesorado impone esta identidad virtual sobre las alumnas que efectivamente demuestran poseer una capacidad mayor que sus compañeros en el aprendizaje de matemática, física y química. Sin embargo, son desechadas como prueba del error del prejuicio en función de su carácter excepcional. Lejos de alzarse como las pruebas empíricas que refutan sus conocimientos del alumnado, inevitable consecuencia a nivel epistemológico, en tanto no se acepta que la excepción confirme la regla, las alumnas que se desempeñan con excelencia o aptitud en los cursos científicos, son tomadas como casos especiales. 
Si estas alumnas no refutan la identidad virtual de género es porque se anticipa el fracaso de las mujeres en el aprendizaje científico, y porque la identidad real de estas alumnas, las estigmatizan en tanto las sitúa en una categoría aparte, especial, dado no conforman lo que naturalmente puede, y, por lo tanto, debe hacer la mujer. Hablamos, así, de prejuicios, de anticipaciones y expectativas, justamente porque los docentes estudiados no actúan en relación a enfoques igualitarios sino a una doxa fundada en el imaginario social, de una sociedad bajo dominación masculina.

Ahora bien, una vez que en el imaginario de los profesores actúa el prejuicio sobre mujeres, también se anticipa y espera lo que sea el hombre. Este es asumido como carente de emociones, de sensibilidad, de madurez emocional.

De este modo, en la enseñanza media se produce la mayor discriminación en contra de ambos sexos-géneros en tanto se naturalizan atributos culturales de lo masculino y de lo femenino. Esta situación incide (junto con otros factores, por supuesto) en la decisión del alumnado sobre qué hacer al egresar y enfrentar la vida adulta. Desde este contexto, y como lo ha demostrado la investigación presente, no sorprende que sea a partir de la enseñanza superior, que las estadísticas nos presenten un panorama donde no solamente las mujeres se auto-excluyen de las carreras universitarias científicas, privilegiando las humanísticas. Así, la identidad virtual de género determina la vida adulta de las niñas y limitan sus oportunidades y opciones. Pocas son las que no actúan desde las expectativas de sus mayores, entre ellos, sus docentes y la cultura dominante.

Creemos que con este estudio, podemos comprender cómo la reproducción de la identidad virtual de género emerge en cada una de las situaciones descritas, en tanto recordamos que la identidad de género apunta a la percepción personal y subjetiva que las (los) estudiantes poseen sobre si mismos en relación a sentirse mujeres $u$ hombres, es entonces en esta instancia, en donde vemos que la sala, la escuela, colabora muchas veces en forma no consciente, a una socialización que no hace otra cosa que reproducir estereotipos sexuales acostumbrados, habituados, contribuyendo a robustecer estas conductas reproductivas discriminatorias. Es sin duda, la escuela la que estaría indicando con su poder, lo que resulta genuino aprender e inconscientemente reproducir, recordemos que los roles de género se asimilan, esto para algunos teóricos significa se despliegan mentalmente en edad temprana para luego reproducirse de la manera que se ha asimilado.

Importa entonces es enfatizar, que en el trabajo de Bourdieu y Passeron (1996) respecto del sistema de enseñanza como reproducción, la variable fundamental era la de 
clase social. En otras palabras, la escuela y la acción pedagógica ponen en funcionamiento los procesos de traspaso de los herencias simbólicos. La escuela, por tanto, reproduce las relaciones de clase social: "la escuela, al sancionar estas diferencias como si fueran puramente escolares, contribuye al mismo tiempo a reproducir la estratificación social y a legitimarla asegurando su interiorización y persuadiendo a los individuos que esta no es social, sino natural" (Bourdieu y Passeron, 1996, p. 17)

De esta manera, el espacio escolar funciona como herramienta "naturalizadora" de las diferencias de clase social, así como también de las diferencias de género. El contexto escolar constituye uno de los espacios que más poderosamente influye en la construcción de la identidad personal de hombres y de mujeres, y de su futuro proyecto de vida (Flores, 2008). Las diferencias entre hombres y mujeres en los resultados de la educación, se deben a que la escuela socializa a niños y niñas no sólo reproduciendo los estereotipos sexuales tradicionales, sino que además contribuyendo a reforzarlos. Así la acción pedagógica de los docentes en el aula, corresponde a la principal forma o práctica a través de la cual la educación reproduce y se convierte en un agente que contribuye a reproducir el orden social. Esta acción se lleva a cabo de una manera específica a través de la potestad y autoridad que sostiene a los profesores (as) y que provine del patronato legítimo, que es la escuela.

Al fin de esta investigación, queda la idea de que aún hay mucho por hacer. Resulta evidente la urgencia de un trabajo de sensibilización y concientización hacia el tema de género. El profesorado debe realizar una auto-crítica de las inconscientes identidades virtuales de género que los llevan a situación de estigmatización. Curiosamente, este proceso es dual: por un lado, se estigmatiza al alumnado según su sexo-género, y, por el otro, el cuerpo docente es estigmatizado por el alumnado. Si bien esta ida y venida empodera al alumnado en la relación asimétrica que constituye la enseñanza-aprendizaje en la enseñanza media, ciertamente resultan ser una resistencia a un poder.

\section{Referencias}

Andrade G., Miguel y Miranda J., Christian. (2001) Ciudadanía y etnicidad: análisis semiótico de las representaciones sociales del concepto de ciudadanía en profesores mapuches y citadinos de educación general en Chile. Revista Profesional Docente, 1(1), 47-78.

Bourdieu, Pierre y Passeron, Jean Claude. (1996). La reproducción, elementos para una teoría del sistema de enseñanza. Barcelona, España: Editorial Laia. Recuperado de http://www.bsolot.info/wp-content/pdf/Bourdieu Pierre\%20\%20La reproduccion Teoria del sistema de ense\%C3\%B1anza.pdf 
Bourdieu, Pierre. (2000). La dominación masculina (traducción de Joaquín Jordá). España: Editorial Anagrama. Recuperado de http://csociales.fmoues.edu.sv/files/La-dominacion$\underline{\text { masculina.pdf }}$

Canales, Manuel. (2009). Metodologías de la Investigación Social. Introducción a los oficios. Santiago de Chile: Editorial LOM.

CEPAL (Comisión Económica para América Latina y el Caribe). (2011). Anuario Estadístico de América Latina y el Caribe. Santiago, Chile: Editorial Naciones Unidas. Recuperado de http://www10.iadb.org/intal/intalcdi/PE/2012/09632.pdf

Dirección del Trabajo. (2011) El derecho a ganar lo mismo. Ley 20.348: Igualdad de remuneraciones entre hombres y mujeres (Temas Laborales $\mathrm{N}^{\circ} 27$ ). Santiago de Chile. Recuperado de http://www.dt.gob.cl/documentacion/1612/articles-99443 recurso 1.pdf

Flores, Raquel. (2005). Violencia de género en la escuela: sus efectos en la identidad, en la autoestima y en el proyecto de vida. Revista Iberoamericana de Educación, (38), 6786. Recuperado de http://www.captel.com.ar/downloads/2406091631 violencia\%20de\%20genero\%20en\% 20la\%20escuela raquel\%20flores\%20bernal.pdf]

Goffman, Erving. (2006). Estigma: La Identidad deteriorada. Buenos Aires: Editorial Amorrortu.

Greimas, Algirdas Julien. (1973). Semántica Estructural. Madrid, España: Editorial Gredos, Tercera Edición.

Greimas, Algirdas Julien. (1976). La semiótica del texto: ejercicios prácticos: Análisis de un cuento de Maupassant. Madrid, España: Editorial Paidós.

Husserl, Edmund. (1962). Introducción general a la fenomenología pura. Nueva York, Estados Unidos: Collier.

Instituto Nacional de Estadísticas (INE). (2007) Encuesta Estructural de Remuneraciones, Costo de Mano de Obra y de Empleo: Boletín Informativo. Santiago de Chile: INE.

Instituto Nacional de la Juventud (INJUV), Ministerio de Desarrollo Social. Gobierno de Chile. (2012). $7^{\circ}$ Encuesta Nacional de Juventud. Recuperado de http://www.injuv.gob.cl/portal/

Kries, Sara. (1993). Aportes de la educación informal a una educación emancipadora en Educación y género: una propuesta emancipadora. Chile: Ediciones La Morada/Ministerio de Educación.

Lamas, Marta (2000). La antropología feminista y las categorías de género. México: Editorial PUEG.

PNUD (Programa de las Naciones Unidad para el Desarrollo). (2010) Desarrollo Humano en Chile. Género: los desafíos de la igualdad. Santiago de Chile. [Versión digital pdf]. 
Recuperado de http://estudios.sernam.cl/documentos/?eMTY3NDAyNA==Informe de Desarrollo Humano. Genero: Los desafios de la igualdad

Reveco, Ofelia. (2011). Currículum y género en la Educación. Revista Latinoamericana de Educación Inclusiva, Vol. $5\left(\mathrm{~N}^{\circ} 1\right)$. Red Iberoamericana de Investigación Sobre Cambio y Eficiencia Escolar. [Versión digital pdf] Recuperado de http://www.rinace.net/rlei/numeros/vol5-num1/art1.pdf.

Servicio de Información de Educación Superior (SIES), División de Educación Superior, Ministerio de Educación. (2012). Informe Matrícula 2012. Recuperado de http://www.mifuturo.cl/images/Informes sies/Matricula/informe matricula2012.pdf

Servicio Nacional de la Mujer (SERNAM), Chile. (s.f.). Servicio Nacional de la Mujer. Recuperado de http://portal.sernam.cl/?m=programa\&i=2

Silva Donoso, María de la Luz. (1989). Encuesta Nacional de Empleo. Santiago, Chile: Programa de la Mujer, Ministerio de Educación e INE. 